\title{
Development of Human Vectored Brucellosis Vaccine Formulation: Assessment of Safety and Protectiveness of Influenza Viral Vectors Expressing Brucella Immunodominant Proteins in Mice and Guinea Pigs
}

\author{
Dina Bugybayeva $\mathbb{D}^{\mathbb{1}},{ }^{1}$ Sholpan Ryskeldinova ${ }^{\mathbb{D}}{ }^{1}{ }^{1}$ Nadezhda Zinina $\mathbb{D}^{\mathrm{D}}{ }^{1}$ \\ Makhpal Sarmykova $\mathbb{D},{ }^{1}$ Nurika Assanzhanova $\mathbb{D},{ }^{1}$ Zhailaubay Kydyrbayev $\mathbb{D},{ }^{1}$ \\ and Kaissar Tabynov $\mathbb{1 D}^{2,3}$ \\ ${ }^{1}$ Research Institute for Biological Safety Problems, 15 Momushuly, Gvardeyskiy 080409, Kazakhstan \\ ${ }^{2}$ Kazakh National Agrarian University, 8 Abay Avenue, Almaty 050010, Kazakhstan \\ ${ }^{3}$ Research Institute of Cardiology and Internal Medicine, 120 Aiteke bi, Almaty 050000, Kazakhstan \\ Correspondence should be addressed to Zhailaubay Kydyrbayev; kydyr2@mail.ru and Kaissar Tabynov; tabynov_81@mail.ru
}

Received 1 July 2020; Revised 10 September 2020; Accepted 15 October 2020; Published 20 November 2020

Academic Editor: Maxim Golovkin

Copyright (c) 2020 Dina Bugybayeva et al. This is an open access article distributed under the Creative Commons Attribution License, which permits unrestricted use, distribution, and reproduction in any medium, provided the original work is properly cited.

\begin{abstract}
In this paper, we first used recombinant influenza viral vector (rIVV) subtype H5N1 expressing from the open reading frame of NS1 80 and NS1 124 amino acids of Brucella outer membrane proteins (Omp) 16 and 19, ribosomal L7/L12, and Cu-Zn superoxide dismutase (SOD) proteins to develop a human brucellosis vaccine. We made 18 combinations of IVVs in mono-, bi-, and tetravalent vaccine formulations and tested them on mice to select the safest and most effective vaccine samples. Then, the most effective vaccine candidates were further tested on guinea pigs. Safety of the rIVV-based vaccine candidate was evaluated by a mouse weight-gain test. Mice and guinea pigs were challenged with the virulent strain $B$. melitensis $16 \mathrm{M}$. The protective effect of the rIVV-based vaccine candidate was assessed by quantitation of Brucella colonization in tissues and organs of challenged animals. All vaccine formulations were safe in mice. Tested vaccine formulations, as well as the commercial $B$. melitensis Rev.1 vaccine, have been found to protect mice from B. melitensis $16 \mathrm{M}$ infection within the range of 1.6 to $2.97 \log _{10}$ units $(P<0.05)$. Tetravalent vaccine formulations from the position of NS1 80 amino acids $(0.2 \pm 0.4)$, as well as the commercial $B$. melitensis Rev.1 vaccine $(1.2 \pm 2.6)$, have been found to protect guinea pigs from $B$. melitensis $16 \mathrm{M}$ infection at a significant level $(P<0.05)$. Thus, tetravalent vaccine formulation Flu-NS1-80-Omp16+Flu-NS1-80-L7/L12+Flu-NS1-80-Omp19+Flu-NS1-80-SOD was chosen as a potential vaccine candidate for further development of an effective human vaccine against brucellosis. These results show a promising future for the development of a safe human vaccine against brucellosis based on rIVVs.
\end{abstract}

\section{Introduction}

Brucellosis is a zoonotic disease that is transmitted between species from animals to humans and is one of the most common infectious diseases in the world, with prevalence in developing countries. Annually, more than 500,000 new cases of human brucellosis are reported [1], and the main pathogenic Brucella species are B. abortus, B. melitensis, and B. suis [2]. Worldwide, B. melitensis is the most prevalent and virulent species causing severe infections in humans [3].

Due to economic and public health consequences of brucellosis in developing countries, efforts have been made to eradicate the disease through vaccination of the livestock sector, as high incidence of the disease in the human population is largely related to the high persistence level of the brucellosis infection in livestock [4]. 
Various vaccine strategies have been made to develop effective brucellosis vaccines with the majority of them intended for human use [5-13]. In particular, vector-based systems on the platform of attenuated viruses (Semliki Forest virus) or bacteria (Ochrobactrum anthropi, Yersinia enterocolitica, and Escherichia coli) have been developed and tested as vaccine candidates against brucellosis, including the presenting recombinant of Brucella proteins as $\mathrm{Cu}-\mathrm{Zn} \mathrm{SOD,} \mathrm{L7/L12,}$ and Omp19 [14-21]. These vector platforms were tested predominantly in mouse models and induced vigorous Th1-type immune responses. In the majority of studies, protection was not confirmed against a challenge with virulent bacterial strain.

Brucella antigens, such as outer membrane proteins (Omp) 16 and 19, ribosomal L7/L12, and Cu-Zn superoxide dismutase (SOD), are inducing a strong cell-mediated response required to clear the infection [22-24]. However, a protective effect of a single epitope of immunization versus a mixed epitope vaccination is not very well understood yet.

The Brucella recombinant influenza viral vector- (IVV-) based vaccine Flu-BA has been developed previously and demonstrated efficacy in bovine comparable with the commercial B. abortus S19 vaccine [25]. The Flu-BA was registered in Kazakhstan in 2019 for vaccination of cattle against B. abortus infection. Bovine species, in general, are not a very susceptible host for influenza A viruses [26]. However, the influenza viral constructs can be more effective in developing a vaccine against human brucellosis, because influenza $A$ is a frequent infection of humans. We believe that rIVV based on subtype $\mathrm{H} 5 \mathrm{~N} 1$ has more potential as a vaccine vector due to the lack of preexisting immunity to $\mathrm{H} 5 \mathrm{~N} 1$ in the general human population [27]. Accordingly, we utilized the rIVV of the H5N1 subtype inserted with Brucella antigens to develop a novel vaccine candidate against human brucellosis.

Thus, the purpose of this study was to select the most optimal vaccine constructs among the safest and protective rIVV expressing Brucella immonodominant proteins Omp16, Omp19, L7/L12, or SOD as a potential candidate for human vaccine development.

\section{Materials and Methods}

2.1. Generation of Virus Constructs and Preparation of Vaccine Samples. IVVs were obtained with the standard reverse genetic method utilizing 8 bidirectional plasmids pHW2000 [28]. In this study, we used eight monovalent vaccine constructs comprising the recombinant influenza $\mathrm{A}$ virus of the subtype H5N1 from the A/chicken/Astana/6/05 strain expressing the Brucella immunodominant proteins L7/L12, Omp16, Omp19, or Cu-Zn SOD containing a sequence of 80 or $124 \mathrm{~N}$-terminal amino acids from the open reading frame (ORF) of the NS1 gene (HSC Development $\mathrm{GmbH}$, Austria). A detailed procedure of rIVV generation is described previously [29]. Briefly, Vero cells were cotransfected by the Lonza Nucleofector ${ }^{\mathrm{TM}}$ (Cologne, Germany) technique with plasmids encoding the PB1, PB2, PA, NP, and $\mathrm{M}$ genes and chimeric NS gene of the A/Puerto Rico/8/34 (H1N1) virus and the hemagglutinin (HA) and neuraminidase (NA) taken from the A/chicken/Astana/6/05
(H5N1) strain. Attenuation of the HA protein sequence of the $\mathrm{H} 5$ virus was provided by exchanging its polybasic cleavage site to one that included a trypsin-dependent sequence. The NS1 gene was modified to insert Brucella sequences. A sequence of 80 or $124 \mathrm{~N}$-terminal amino acids from the NS protein paired with a sequence of Brucella proteins.

The obtained influenza vectors (Flu-NS1-80-Omp16, Flu-NS1-80-L7/L12, Flu-NS1-80-Omp19, Flu-NS1-80-SOD, Flu-NS1-124-Omp16, Flu-NS1-124-L7/L12, Flu-NS1-124Omp19, and Flu-NS1-124-SOD) were then used for producing vaccine samples in 10-day-old embryonated chicken eggs (CE) as reported previously [29]. The insertion of referred Brucella proteins in the NS1 gene was confirmed by reverse transcription polymerase chain reaction (RT-PCR) Figure S1. Obtained allantoic fluid containing IVV inserted with various Brucella antigenic genes used as a mono formulation or pooled together in a $1: 1$ or $1: 1: 1: 1$ ratio to obtain overall $18 \mathrm{rIVV}$ mono or combinations of bivalent and tetravalent formulations.

2.2. Bacterial Strains. The virulent strain of B. melitensis $16 \mathrm{M}$, obtained from the Research Institute for Biological Safety Problem's (RIBSP) collection of microorganisms, was used in this study. The bacterial cells were cultured in Brucella base agar (Himedia Laboratories, India) under aerobic conditions at $37^{\circ} \mathrm{C}$. All laboratory experiments with live Brucella cells were conducted in biosafety level (BSL) 3. Challenged mice and guinea pigs were kept in animal BSL 3 facility.

2.3. Bioethics and Animal Groups. This study was carried out in accordance with the recommendations of the national and international guidelines on animal care and use. The study protocol was approved by the IACUC of the RIBSP (approval no. 0418/04). Animals were housed in a 12 light/12 dark cycle in cages under controlled environmental conditions and were fed ad libitum with standard rodent diet and had no water restrictions. Experimental and control groups of animals were kept in different rooms during the entire experiment. This study used male BALB/c mice (Charles River) aged 5-7 weeks old weighing 20-25 g and conventional bred female guinea pigs weighing 300-350 g (National Center for Expertise of Drugs, Medical Products and Equipment, Kazakhstan). By the randomization method, mice were divided into 20 groups ( $n=12$ per group): 18 experimental prime-boost groups, one negative PBS (phosphate-buffered saline) control group, and one positive control group. The guinea pigs were divided into 7 groups ( $n=5$ per group): five experimental prime-boost groups, one negative (PBS) control group, and one positive control group.

2.4. Immunization of Mice. Groups of mice in experimental prime-boost groups ( $n=5$ or $n=12$ per group) for safety or challenge studies were injected peritoneally (i.p.) twice with prepared vector brucellosis vaccine formulations (18 overall) at an interval of 14 or 21 days. After selection of mice within these 18 formulations, 5 vaccine formulations that demonstrated significant results were further tested on guinea pigs with the same prime-boost immunization scheme when rIVV was administered intranasally (i.n.) twice with an 
TABLE 1: Scheme of immunization of animals.

\begin{tabular}{|c|c|c|c|}
\hline Species & Viral construct & $\begin{array}{l}\text { Prime vaccination dose } \\
\left(\log _{10} \mathrm{EID}_{50} / \text { animal }\right)\end{array}$ & $\begin{array}{c}\text { Booster vaccination dose } \\
\left(\log _{10} \mathrm{EID}_{50} / \text { animal }\right)\end{array}$ \\
\hline \multirow{9}{*}{$\begin{array}{l}\text { Mice } \\
\text { i.p. }\end{array}$} & Monovalent vaccine formulation (VF) & & \\
\hline & (1) Flu-NS1-80-Omp16 & (1) 6.14 & (1) 6.22 \\
\hline & (2) Flu-NS1-80-L7/L12 & (2) 6.06 & (2) 6.14 \\
\hline & (3) Flu-NS1-80-Omp19 & (3) 6.64 & (3) 6.56 \\
\hline & (4) Flu-NS1-80-SOD & (4) 6.56 & (4) 6.22 \\
\hline & (5) Flu-NS1-124-Omp16 & (5) 6.64 & (5) 6.56 \\
\hline & (6) Flu-NS1-124-L7/L12 & (6) 6.69 & (6) 6.64 \\
\hline & (7) Flu-NS1-124-Omp19 & (7) 6.22 & (7) 6.31 \\
\hline & (8) Flu-NS1-124-SOD & $(8) 6.31$ & $(8) 6.56$ \\
\hline \multirow{9}{*}{$\begin{array}{l}\text { Mice } \\
\text { i.p. }\end{array}$} & Bivalent VF & & \\
\hline & (9) Flu-NS1-80-Omp16+Flu-NS1-80-L7/L12 & (9) $5.84+5.76$ & (9) $5.92+5.84$ \\
\hline & (10) Flu-NS1-80-Omp19+Flu-NS1-80-SOD & (10) $6.34+6.26$ & (10) $6.26+5.92$ \\
\hline & (11) Flu-NS1-80-Omp16+Flu-NS1-80-Omp19 & (11) $5.84+6.34$ & (11) $5.92+6.26$ \\
\hline & (12) Flu-NS1-80-L7/L12+Flu-NS1-80-SOD & (12) $5.76+6.26$ & (12) $5.84+5.92$ \\
\hline & (13) Flu-NS1-124-Omp16+Flu-NS1-124-L7/L12 & (13) $6.34+6.39$ & (13) $6.26+6.34$ \\
\hline & (14) Flu-NS1-124-Omp19+Flu-NS1-124-SOD & (14) $5.92+6.01$ & (14) $6.01+6.26$ \\
\hline & (15) Flu-NS1-124-Omp16+Flu-NS1-124-Omp19 & (15) $6.34+5.92$ & (15) $6.26+6.01$ \\
\hline & (16) Flu-NS1-124-L7/L12+Flu-NS1-124-SOD & (16) $6.39+6.01$ & (16) $6.34+6.26$ \\
\hline \multirow{3}{*}{$\begin{array}{l}\text { Mice } \\
\text { i.p. }\end{array}$} & Tetravalent VF & & \\
\hline & $\begin{array}{l}\text { (17) Flu-NS1-80-Omp16+Flu-NS1-80-L7/L12+ } \\
\text { Flu-NS1-80-Omp19+Flu-NS1-80-SOD }\end{array}$ & (17) $5.54+5.46+6.04+5.96$ & (17) $5.62+5.54+5.96+5.62$ \\
\hline & $\begin{array}{l}\text { (18) Flu-NS1-124-Omp16+Flu-NS1-124-L7/L12+ } \\
\text { Flu-NS1-124-Omp19+Flu-NS1-124-SOD }\end{array}$ & (18) $6.04+6.09+5.62+5.71$ & (18) $5.96+6.04+5.71+5.96$ \\
\hline \multirow{5}{*}{$\begin{array}{l}\text { Guinea pigs* } \\
\text { i.n. }\end{array}$} & (1) Flu-NS1-80-Omp16 & (1) 6.75 & (1) 6.83 \\
\hline & (8) Flu-NS1-124-SOD & (8) 6.92 & (8) 7.04 \\
\hline & (15) Flu-NS1-124-Omp16+Flu-NS1-124-Omp19 & (15) $6.95+6.53$ & (15) $6.87+6.62$ \\
\hline & $\begin{array}{l}\text { (17) Flu-NS1-80-Omp16+Flu-NS1-80-L7/L12 } \\
\text { +Flu-NS1-80-Omp19+Flu-NS1-80-SOD }\end{array}$ & (17) $6.14+6.06+6.64+6.56$ & (17) $6.22+6.14+6.56+6.22$ \\
\hline & $\begin{array}{l}\text { (18) Flu-NS1-124-Omp16+Flu-NS1-124-L7/L12+ } \\
\text { Flu-NS1-124-Omp19+Flu-NS1-124-SOD }\end{array}$ & (18) $6.64+6.69+6.22+6.31$ & (18) $6.56+6.64+6.31+6.56$ \\
\hline
\end{tabular}

Table note: number of animals per viral construct vaccine formulation for mice was 5 or 12 and for guinea pigs was 5 per group. The amounts of substance for mice via intraperitoneal (i.p.) were $500 \mu \mathrm{l}$, and for guinea pigs, intranasal (i.n.) immunization was $200 \mu \mathrm{l}$ in both nostrils. ${ }^{*}$ After the evaluation of protective efficacy of 18 vaccine formulations in mice, the 5 most protective vaccine formulations were selected and then tested in guinea pigs.

interval of 21 days between vaccinations. The animal immunization scheme is presented in Table 1. Mice and guinea pigs from the positive control groups were injected subcutaneously (s.c.) with the B. melitensis Rev.1 (Antigen LLP, Kazakhstan) only once at a dose of $6.0 \log _{10}$ CFU/animal. Mice and guinea pigs of the negative control groups s.c. injected with $200 \mu \mathrm{l}$ of PBS.

2.5. Assessment of Mono-, Bi-, and Tetravalent Vaccine Formulation Safety. To assess the safety of vaccine samples (or their level of attenuation), vaccinated mouse body weight changes were monitored daily for 28 days after both prime and booster vaccinations with viral constructs or $B$. melitensis Rev.1 (vaccination was provided only once) and compared to the negative (PBS) control group. General observation for safety was evaluated by animal survival and animals' general condition, behavior, and dynamics of body weight change.

2.6. Assessment of the Vaccine Formulation's Protectiveness in Mice and Guinea Pigs. In order to evaluate vaccine formulation's protectiveness, mice from the experimental vaccine groups ( $n=5$ per group) and PBS injected control group $(n=5)$ were challenged i.p. with the virulent strain of $B$. melitensis $16 \mathrm{M}$ in a dose of $6.0 \log _{10} \mathrm{CFU} /$ animal on 21 days after the boost vaccination. Mice in the positive control group $(n=5)$ were immunized with the vaccine $B$. melitensis Rev.1 and challenged on day 42. On day 14 postchallenge, all animals were sacrificed by $\mathrm{CO}_{2}$ asphyxiation to collect spleen tissues aseptically for bacteriological studies. The spleen from each animal was harvested and homogenized 
in $5 \mathrm{ml}$ of $0.1 \%$ Triton-PBS, and $100 \mu \mathrm{l}$ of 10 -fold serial dilutions of spleen suspension was plated in triplicate onto Brucella base agar (HiMedia Laboratories, India) plates. Plates were incubated at $37^{\circ} \mathrm{C}$ for 2 weeks, and the number of bacterial colony growth was counted periodically by performing standard plate counts to determine the concentration of bacteria (CFU/tissue) in spleens. An animal was considered to be infected if one or more Brucella colonies were present in the cultures. The protective effect of the vaccine samples was assessed by comparing the degree of spleen infection of vaccinated experimental and reference control groups and unvaccinated control group after virulence challenge with B. melitensis $16 \mathrm{M}$ (expressed as $\log _{10} \mathrm{CFU} / \mathrm{g}$ of tissue protection unit).

After studying the protective efficacy of vaccine formulations, the five most protective vaccine formulations were assessed in the guinea pig model. The challenge study for guinea pigs was similar as what was done for mice, and it was consistent based on route of infection, grouping of animals, preparing suspension from animal organs, bacteria growing conditions, and counting of colonies. Twenty-one days after the boost vaccination, guinea pigs from the experimental groups $(n=25)$, PBS control $(n=5)$, and positive control $(n=5)$ (on day 42 after prime vaccination with vaccine B. melitensis Rev.1) groups were challenged s.c. with the virulent strain of $B$. melitensis $16 \mathrm{M}$ in a dose of 1.3 $\log _{10}$ CFU/animal. Thirty days after challenge, animals from all groups were euthanized and aseptically dissected to collect the following tissues: retropharyngeal, lower cervical, right and left inguinal lymph nodes, liver, spleen, and bone marrow. The results of the bacteriological assessment were evaluated from three parameters, including vaccination efficacy (expressed in \%) determined as the number of animals from which no colonies were isolated, infection index (number of tissues from which Brucella were isolated in), and protective efficacy evaluated as the degree of Brucella colonization in organs and lymph nodes expressed as $\log _{10} \mathrm{CFU} / \mathrm{g}$ of tissue.

2.7. Statistical Analysis. The safety of vaccine formulations was analyzed using one-way ANOVA (Dunnett's multiple comparison test). Protection of vaccines and index of infection data were analyzed with a one-way ANOVA (Tukey's multiple comparison test) and two-way ANOVA (Sidak's multiple comparison test), respectively. The variance in protective efficacy of animal groups was compared by one-sided Fisher exact test. $P$ values $<0.05$ were considered significant. Means are reported with standard errors (SEM) and 95\% confidence interval. Statistical analysis was performed with GraphPad Prism Software, version 6.0 (GraphPad Software Inc., La Jolla, CA, USA). The experiments have been repeated, and the results were reproducible.

\section{Results}

3.1. Assessment of Various Vaccine Formulations' Safety in Mice. The safety or degree of attenuation of various vaccine formulations, comprising mono-, bi-, or tetravalent rIVV, were determined in mice in comparison with the positive (B. melitensis $16 \mathrm{M}$ ) and negative (PBS) control groups. It was found that all vaccine samples, including $B$. melitensis Rev.1, were safe in mice after i.p. injection. No animal death or disease sign was observed in any group by the end of the observation period. Overall, condition of animals both in the control and experimental groups was moderate, in terms of physical activity, appetite, and general outward condition.

Assessment of percent change of body weight over 28 days upon prime or boost immunizations showed an increase in the animals' body weight for all type of viral construct (Figure 1). By the end of the observation period, the weight of mice in the experimental groups has been increased by $19-29 \%$ or $3.8-5.8 \mathrm{~g}$, which was similar to the control group $-28 \%$ or $4.7 \mathrm{~g}$. No group was significantly different in mean of body weight from the PBS-treated control group $(P>0.5)$.

It should be noted that examination of the Brucella proteins in the NS1 gene by the RT-PCR confirmed that all the viral constructs retained their corresponding Brucella inserts upon producing rIVV in CE.

3.2. Protectiveness of Vaccine Formulations in Mice against B. melitensis $16 \mathrm{M}$ Infection. This experiment was conducted to assess protective efficacy of 18 mono-, bi-, or tetravalent vaccine formulations first in a mouse model to determine the most protective vaccine formulations against $B$. melitensis $16 \mathrm{M}$ infection and to compare to the reference $B$. melitensis Rev.1 vaccine group or PBS control group. The protective activity of the vaccines was evaluated by bacterial load or virulent Brucella bacteria colonization in spleens of vaccinated and nonvaccinated animals. The results of the bacteriology study after the virulence challenge showed that mono-, bi-, or tetravalent vaccine formulations as well as B. melitensis Rev.1 provided protection between 1.6 and $2.79 \log _{10}$ units. Compared to the PBS unvaccinated control group, all vaccine formulations as well as the commercial vaccine provided protection of mice from $B$. melitensis $16 \mathrm{M}$ infection on rates of virulent strain colonization in challenged animal spleens (Table 2).

Significant protection in comparison with the PBS control group was achieved in the following 5 groups: (1) 80-Omp16, (8) 124-Cu-Zn-SOD, (13) 80-Omp16+L7/L12+ Omp19+Cu-Zn-SOD, (16)124-Omp16+Omp19, and (18) 124-Omp16+L7/L12+Omp19+Cu-Zn-SOD $(P<0.02)$ as well as in the positive control group vaccinated with $B$. melitensis Rev.1 $(P<0.02)$. By the result of this study, the five most protective vaccine formulations that showed significant results in mice were continued in guinea pigs to confirm an efficacy of selected vaccine formulations.

3.3. Assessing of Protection of Five Vaccine Formulations in Guinea Pigs. From the 18 vaccine formulations assessed in mice, the five most protective ones were further investigated in a guinea pig model. The protective efficacy of viral vectors expressing immunodominant Brucella proteins Omp16, L7/L12, Omp19, and SOD at mono-, bi-, or tetravalent vaccine formulations was compared to that of a commercial vaccine B. melitensis Rev.1 and PBS and assessed by parameters, such as colonization of the virulent strain of $B$. melitensis $16 \mathrm{M}$ in the lymph nodes and organs of the vaccinated and 


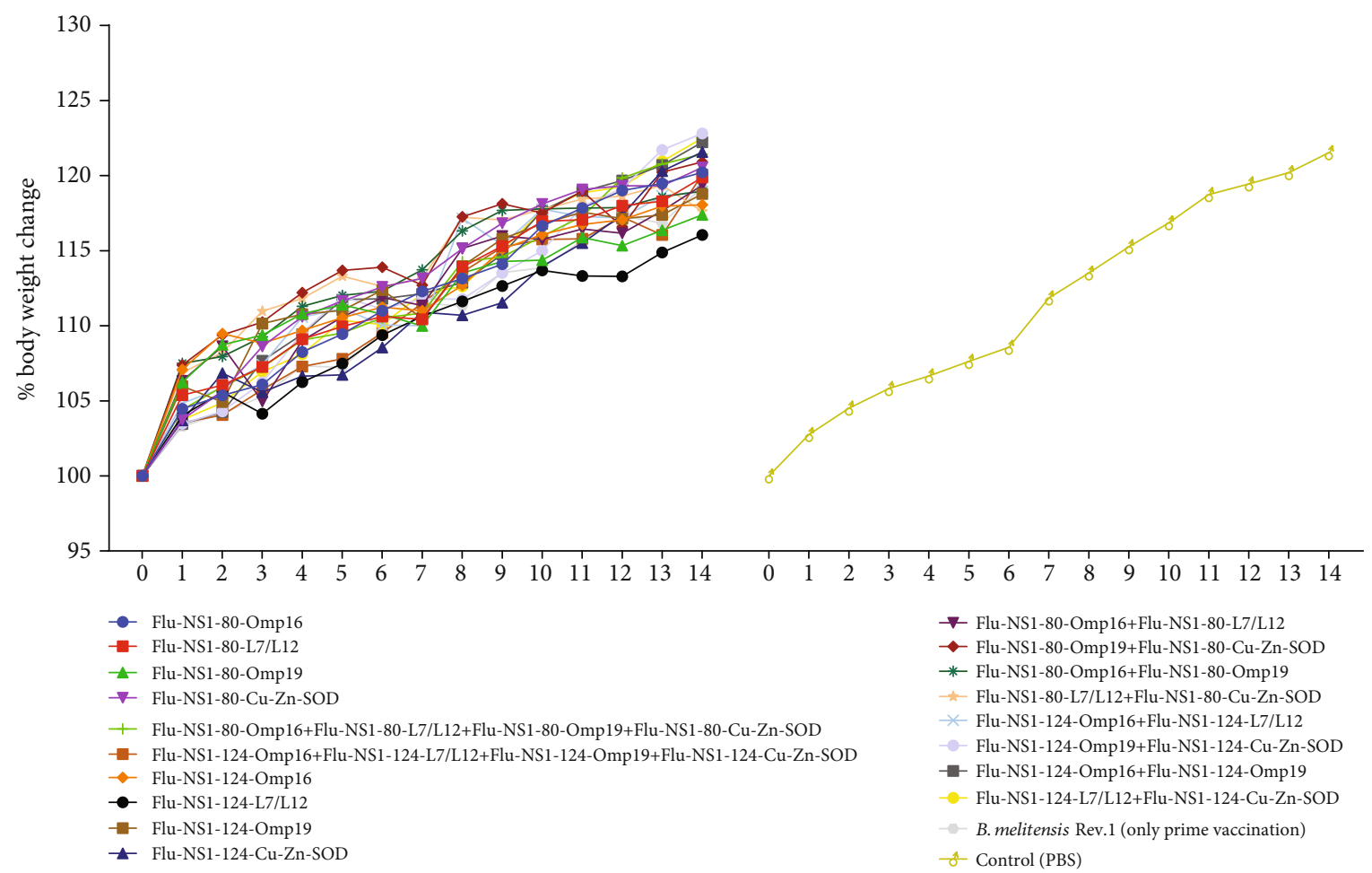

(a)

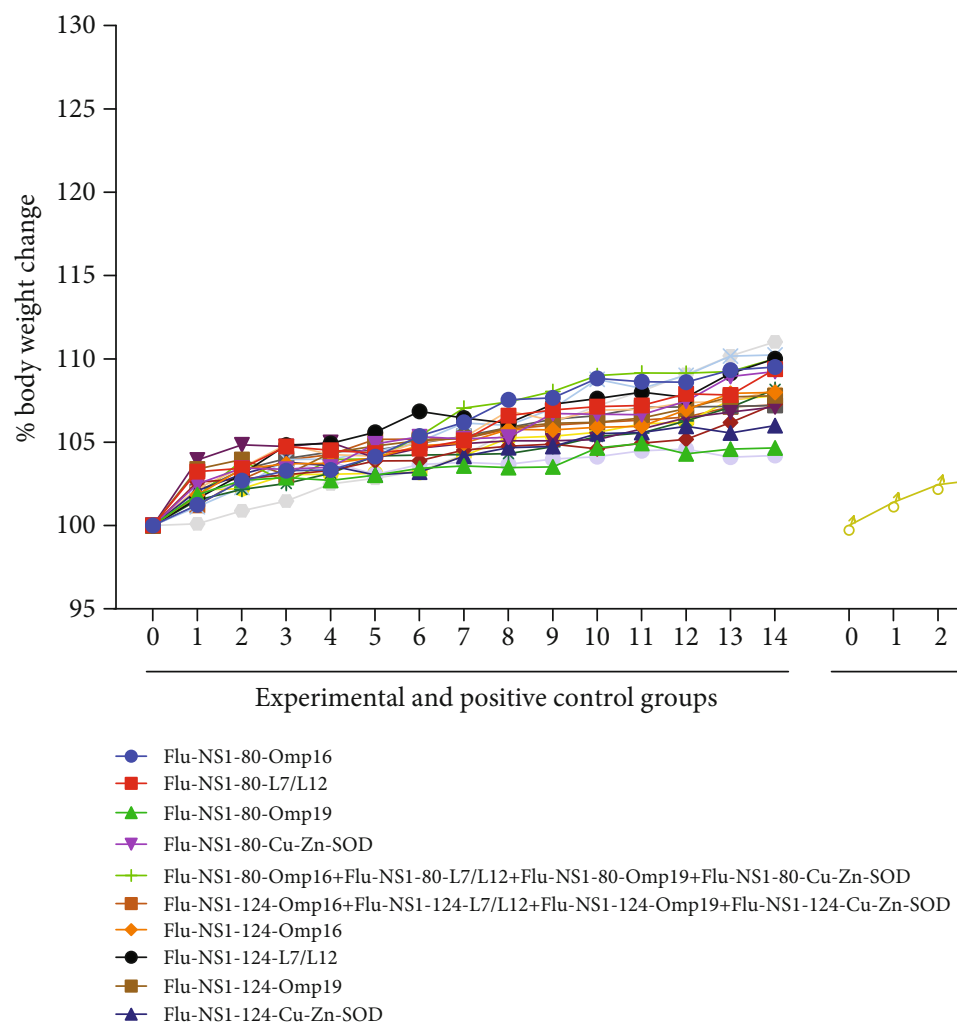

(b)

Figure 1: Percentages of body weight change of mice after prime-booster immunization. Percentages of body weight change of mice in experimental and control (after single vaccination with B. melitensis Rev.1 or administration of PBS) groups recorded daily 28 days after prime (a) and booster (b) immunization with mono-, bi-, or tetravalent formulations of rIVVs. Statistical analysis was performed with one-way ANOVA followed by Dunnett's multiple comparison test showed that during the 28 days, body weight measurement between the PBS control and vaccinated groups was not significant. $P<0.05$ values were considered significant. 
TABLE 2: Level of protective efficacy of vaccines assessed by the isolation rate of Brucella from the spleens of mice challenged with the virulent strain B. melitensis $16 \mathrm{M}$.

\begin{tabular}{|c|c|c|c|c|c|c|}
\hline Groups & Vaccine samples & $\begin{array}{l}\text { No. of } \\
\text { animals }\end{array}$ & $\begin{array}{l}\text { Brucella titer, } \log _{10} \mathrm{CFU} / \mathrm{g} \text { spleen } \\
(\text { mean } \pm \mathrm{SE})\end{array}$ & $\begin{array}{l}\text { Protection unit } \\
\qquad\left(\log _{10}\right)^{*}\end{array}$ & $\begin{array}{l}\text { Signifi } \\
\text { contro } \\
(+) \\
\text { control }\end{array}$ & $\begin{array}{l}\text { nce to } \\
\text { group } \\
(-) \\
\text { control }\end{array}$ \\
\hline 1 & 80-Omp16 & 5 & $3.08 \pm 0.86$ & $2.79^{*}$ & $>0.05$ & $<0.05$ \\
\hline 2 & 80-L7/L12 & 5 & $3.7 \pm 0.33$ & 2.17 & $>0.05$ & $>0.05$ \\
\hline 3 & 80-Omp19 & 5 & $3.68 \pm 0.37$ & 2.2 & $>0.05$ & $>0.05$ \\
\hline 4 & 80-SOD & 5 & $3.64 \pm 0.38$ & 2.24 & $>0.05$ & $>0.05$ \\
\hline 5 & 124-Omp16 & 5 & $3.41 \pm 0.84$ & 2.46 & $>0.05$ & $>0.05$ \\
\hline 6 & 124-L7/L12 & 5 & $3.47 \pm 0.55$ & 2.40 & $>0.05$ & $>0.05$ \\
\hline 7 & 124-Omp19 & 5 & $4.1 \pm 0.29$ & 1.78 & $>0.05$ & $>0.05$ \\
\hline 8 & 124-SOD & 5 & $3.2 \pm 0.76$ & $2.60^{*}$ & $>0.05$ & $<0.05$ \\
\hline 9 & 80-Omp16+L7/L12 & 5 & $3.78 \pm 0.41$ & 2.1 & $>0.05$ & $>0.05$ \\
\hline 10 & 80-Omp19+SOD & 5 & $4.18 \pm 0.3$ & 1.7 & $>0.05$ & $>0.05$ \\
\hline 11 & 80-Omp16+Omp19 & 5 & $3.83 \pm 0.39$ & 2.04 & $>0.05$ & $>0.05$ \\
\hline 12 & 80-L7/L12+SOD & 5 & $3.57 \pm 0.28$ & 2.30 & $>0.05$ & $>0.05$ \\
\hline 13 & 80-Omp16+L7/L12+Omp19+SOD & 5 & $3.13 \pm 0.25$ & $2.75^{*}$ & $>0.05$ & $<0.05$ \\
\hline 14 & 124-Omp16+L7/L12 & 5 & $3.49 \pm 0.36$ & 2.38 & $>0.05$ & $>0.05$ \\
\hline 15 & 124-Omp19+SOD & 5 & $3.64 \pm 0.53$ & 2.23 & $>0.05$ & $>0.05$ \\
\hline 16 & 124-Omp16+Omp19 & 5 & $3.18 \pm 0.69$ & $2.69^{*}$ & $>0.05$ & $<0.05$ \\
\hline 17 & 124-L7/L12+SOD & 5 & $4.27 \pm 0.3$ & 1.60 & $>0.05$ & $>0.05$ \\
\hline 18 & 124-Omp16+L7/L12+Omp19+SOD & 5 & $3.17 \pm 0.38$ & $2.70^{*}$ & $>0.05$ & $<0.05$ \\
\hline 19 & B. melitensis Rev.1 & 5 & $3.20 \pm 0.31$ & $2.68^{*}$ & - & $<0.05$ \\
\hline 20 & Control (PBS) & 5 & $5.88 \pm 0.16$ & - & $<0.05$ & - \\
\hline
\end{tabular}

Table legend: ${ }^{*} \log _{10}$ protection units were obtained by subtracting the mean $\log _{10}$ CFU of the control (PBS) group from the mean of $\log _{10}$ CFU for the experimental group and for the positive control group. (+) control: animals vaccinated with B. melitensis Rev.1 commercial vaccine. (-) control: animals inoculated with PBS. *alpha $=0.02-0.01$ vs. PBS control group, B. melitensis Rev.1 vs. vaccine groups. Statistical analysis was performed using a one-way ANOVA (Tukey's multiple comparison test).

unvaccinated animals and index of infection (number of tissues and organs from which Brucella bacteria were isolated). All vaccine formulations and the commercial vaccine provided significant protection $(P<0.01-P<0.0001$ compared to the PBS control group) to the guinea pigs against the virulent strain B. melitensis $16 \mathrm{M}$ to a certain degree, specifically in the number of cultured Brucella in tissues and organs of animals upon the challenge. In animals vaccinated with 80 Omp16+L7/L12+Omp19+SOD (Figure 2(a), average value for the group: $0.01 \log _{10} \mathrm{CFU} / \mathrm{g}$ of tissue) and 124-Omp16 +L7/L12+Omp19+SOD (average value for group: $1.01 \log _{10}$ $\mathrm{CFU} / \mathrm{g}$ of tissue), we found a low degree of Brucella colonization in tissues (for the NS1-80 tetravalent group only in the spleen) in comparison with the PBS control group (2.9 $\log _{10} \mathrm{CFU} / \mathrm{g}$ of tissue).

According to the index of infection (Figure 2(b)), a significant level of protection in comparison with the control challenge group (the infection rate, 100\%) was achieved in the group that was vaccinated with the tetravalent viral construct $(P<0.01$; vaccination efficacy, 80\%) expressing the Brucella
Omp16, L7/L12, Omp19, and SOD proteins fused to the Nterminal 80 amino acids of NS1. It is worth noting that although in the animal group vaccinated with $B$. melitensis Rev.1, the effectiveness of vaccination (Table 3) reached $80 \%(P=0.02)$; however, the index of infection for the $B$. melitensis Rev.1 group was not significantly different from that for the groups vaccinated with the above-mentioned tetravalent viral constructs.

\section{Discussion}

The available commercial vaccines against brucellosis are limited to use just in small ruminants and cattle because of the adverse effect of these vaccines for human use [30]. The search for safe and effective human brucellosis vaccines remains active today, specifically for farmers in endemic places as well as for veterinarians with risks related to the occupational exposure and animal care workers [31]. Numerous candidate vaccines and vaccine strategies against B. abortus have been evaluated in animal models, including 
80-Omp16

80-Omp16+L7/L12+Omp19+SOD
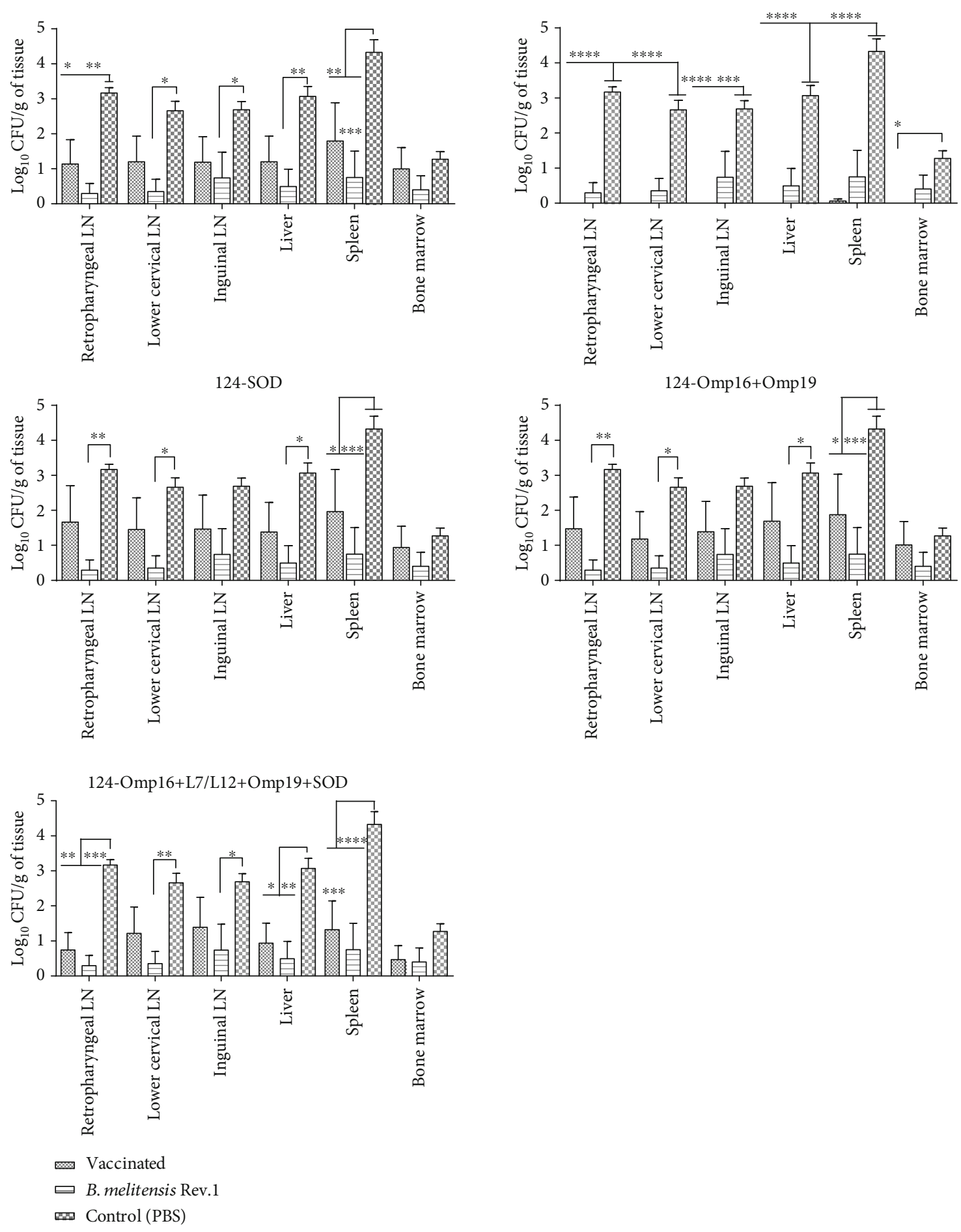

(a)

FIgure 2: Continued. 


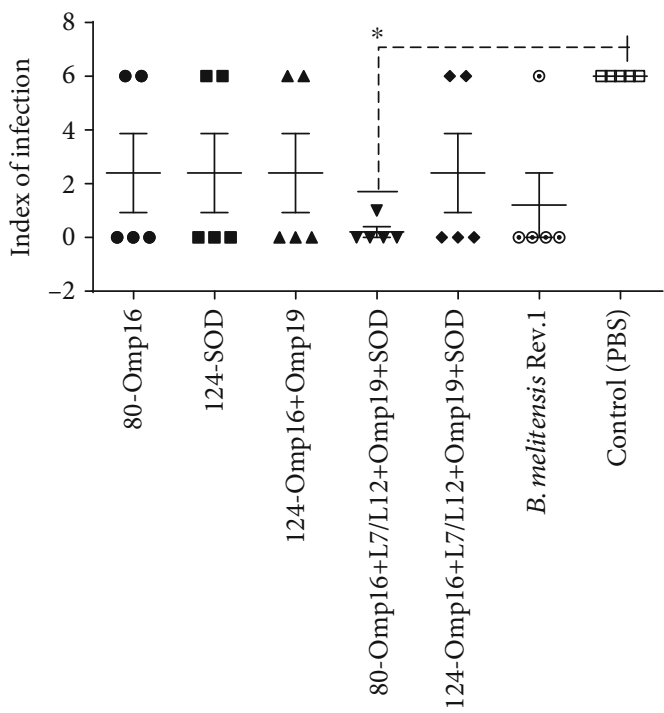

(b)

Figure 2: Protectiveness of vaccine samples in guinea pigs estimated by the amount allocated to Brucella from tissues and organs (a) and index of infection (b). Animals were vaccinated at regime of prime-boost at interval of 21 days with mono-, bi-, or tetravalent vaccine formulations or a single delivery of commercial vaccine B. melitensis Rev.1. Guinea pigs in the negative control group were delivered with PBS. The challenge of animals was performed with virulent strain of B. melitensis $16 \mathrm{M}$ at a dose of $1.3 \log _{10}$ CFU/animal using an s.c. route. Bacteriological evaluation was assessed by counting Brucella colonies in tissues, where data is expressed as $\log _{10}$ CFU/g and the index of infection in animals (the arithmetic mean \pm standard error was given for each group; number of tissues from where Brucella was isolated for each animal). Statistical analysis for (a) was performed using a one-way ANOVA followed by Tukey's multiple comparison test, and for (b) two-way ANOVA followed by Sidak's multiple comparison test. From ${ }^{*} P=0.01$ to $P=0.04$; from ${ }^{* *} P=0.002$ to $P=$ 0.004 ; from ${ }^{* * *} P=0.0002$ to $P=0.0007 ;{ }^{* * *} P<0.0001$.

TABLE 3: Rates of protection in guinea pigs after challenge with the virulent strain B. melitensis 16M.

\begin{tabular}{lccc}
\hline Immunization group & Total animals & Isolation of B. melitensis in animals, $n(\%)$ & $\begin{array}{c}\text { Value }(P)^{*} \\
(-) \text { control }\end{array}$ \\
\hline 80-Omp16 & 5 & $2(40)$ & $>0.05$ \\
80-Omp16+L7/L12+Omp19+SOD & 5 & $1(20)$ & $>0.05$ \\
124-SOD & 5 & $2(40)$ & $>0.05$ \\
124-Omp16+Omp19 & 5 & $2(40)$ & $>0.05$ \\
124-Omp16+L7/L12+Omp19+SOD & 5 & $2(40)$ & $>0.05$ \\
B. melitensis Rev.1 & 5 & $1(20)$ & $>0.05$ \\
Control (PBS) & 5 & $5(100)$ & $>0.05$ \\
\hline
\end{tabular}

Table note: *in comparison with control untreated PBS or B. melitensis Rev.1 groups. Statistical analysis was performed using a one-sided Fisher's exact test. $<0.05$ : $P$ value less than $0.05 ;>0.05: P$ value higher than 0.05 .

DNA vaccine, recombinant subunit peptide, protein, LPS, outer membrane vesicles (OMV), a live vector (viral or bacterial vector-based Brucella vaccines), combinations in prime and boost strategies, and others. Brucella recombinant vaccinia viruses expressing L7/L12, Omp18, and GroEL proteins have been studied in mouse models without substantial protection against Brucella challenge [32-34]. Highly immunogenic constructs have been developed based on an adenoviral vector expressing both p39 and lumazine synthase proteins of B. abortus [35]. However, preexisting immunity to the viral vector could prevent a vaccine from working. To circumvent the problem of preexisting immunity, nonhuman adenovirus vectors or genetically modified adenovirus constructs could be used as the vaccine carrier. Replicationdeficient Semliki Forest virus expressing Brucella translation initiation factor 3 (IF3) and Sod C generated some Th1 response and partial protection in mice $[36,37]$. Influenza viruses expressing Brucella ribosomal proteins L7/L12 and Omp16 produced a long-term protection in pregnant heifers against $B$. abortus infection $[38,39]$. As reported previously, immunization with $B$. abortus recombinant influenza A viruses based on subtypes $\mathrm{H} 5 \mathrm{~N} 1$ or $\mathrm{H} 1 \mathrm{~N} 1$ was protective in cattle [38]. However, bovines are naturally immune to influenza infections. We reasoned if we could generate protection in mouse and guinea pig models using improved Brucella antigen composition in influenza $A$ viruses based on 
subtypes $\mathrm{H} 5 \mathrm{~N} 1$ as vaccine career, then we can potentially use these recombinant vaccines for preclinical and clinical trials in humans.

There is absence or little preexisting immunity against influenza virus A of subtype $\mathrm{H} 5 \mathrm{~N} 1$ in the human population. This is an important conceptual basis and quality of our vaccine vector candidates for future Brucella vaccine development in humans. We used a previously generated backlog of viral constructs, specifically $8 \mathrm{IVV}$ of subtype H5N1 expressing Omp16, L7/L12, Omp19, and Cu-Zn SOD inserted into the NS1 gene region at position 80 or 124 amino acids. From these viral constructs were formed 18 vaccine formulations to characterize their safety and protection in mice and guinea pigs to form the final vaccine formulation. As the vaccine intended for use in humans, placing outbred animals $(\mathrm{BALB} / \mathrm{c}$ mice) and a less homogeneous population of guinea pigs should demonstrate relationship in consistency and performance.

In the present study, we used intraperitoneal (i.p.) inoculation of rIVV in the prime-boost immunization strategy in mice and intranasal (i.n.) administration in the guinea pig model. Animals in the control group have been vaccinated with commercial B. melitensis Rev.1 s.c. by the manufacturer's recommendations. For this study, the influenza A viral vector was generated on the backbone of the NS (chimeric) gene of Puerto Rico/8/34 (H1N1), and the surface genes for hemagglutinin and neuraminidase were taken from A/chicken/Astana/6/05 (H5N1) strain. The PR8 virus is a mouse-adapted virus with efficient replicative properties in mice that could cause an infectious process in mouse lungs and lead to mortality when delivered i.n. [40-43]. Therefore, we used i.p. inoculation of vaccine samples in mice due to the nature of the PR8 virus to cause disease symptoms.

As the influenza virus has tropism to mucosal surfaces, we believe that the optimal way for rIVV immunization is the i.n. route. Bearing in mind that Brucella should be considered as a mucosal pathogen, penetrating the mucosa of the nasal or oral cavities after ingestion, mucosal vaccination is capable of generating protective responses against pathogens at the mucosal site of entry [44].

Safety or attenuation of rIVV conferred by the shortened NS1 gene that facilitates their limited replicative ability [29]. We found that all vaccine formulations were safe, and no animal death or weight decrease was observed in mice. Additionally, after the challenge study in mice, we took five effective vaccine formulations and used them for i.n. immunization of guinea pigs. Importantly, bacteriological studies in mice demonstrated that that rIVVbased vaccine formulations demonstrated a similar level of protection as commercially established vaccine $B$. melitensis Rev.1. These data were reproducible in the guinea pig model as well. Thus, we concluded that rIVV vaccine formulations were as effective as the commercial vaccine. Formulations coding amino acids at positions 80 or 124 , especially tetravalent constructs, in protection studies demonstrated results similar to those of the group vaccinated with the commercial vaccine. In addition, protective efficacy and immunogenicity of the candidate vaccine were established through a standardized challenge study by the ability of an animal model to restrain bacteria in the spleen [45].

To our knowledge, this is the first study to utilize a Brucella recombinant vector coding 80 amino acids at ORF of the NS1 for protective studies in animal models. For development of a human vaccine, NS1-80 may have some advantages. Knowing interferon antagonist properties of the influenza NS1 protein, viral constructs with a length of NS1-80 amino acid size should be less aggressive than NS124 , which has a half-length of the NS1. There is some correlation between length of the NS1 protein and attenuation of the virus in live organisms [46]. Because IVV has a different infectivity based on the length of the NS1 protein, combined use of IVV with different lengths of the NS1 protein (80 and 124) can lead to the interference of individual viruses, which is not observed when using IVV with the same length of NS1 protein.

There are some concerns in general about using IVV of subtype $\mathrm{H} 5 \mathrm{~N} 1$, which is a pathogenic type of influenza virus circulated in birds. Major concerns related to interspecies transmission of the disease from bird to human may cause diseases in the human population. However, in our case, the virus has proven to be attenuated through removal of the proteolytic cleavage site in the HA, and safety of the rIVV is ensured through the shortened NS1 gene, and as the result, they have limiting replicative ability [29]. Another risk related to using rIVV to public health is generation of reassortment between avian $\mathrm{H} 5 \mathrm{~N} 1$ and human influenza viruses that might confer pandemic strains [47]. However, for more than 30 years of using live attenuated cold-adapted influenza virus vaccine for humans, there are no records to date of a new emergence of virulent reassortants [48]. This vaccine candidate is not intended for mass vaccination and will be used for people in the risk groups only. Our previous studies have demonstrated that after repeated passages in CE, the IVV retained its main biological properties, including attenuation, and did not lose Brucella antigen inserts [29], indicating their genetic stability. In addition, the IVV belong to the group of RNA viruses, which facilitates the limiting replicative process that eliminates the risk of integration and longterm persistence.

\section{Conclusions}

Thus, the results of this study demonstrated that recombinant influenza virus subtype A/H5N1 expressing the Brucella L7/L12 or Omp16 or Omp19 or Cu-Zn SOD (SOD) proteins from the open reading frame (ORF) of the NS1 gene in combination with tetravalent formulations is a safe vector, and its protectiveness against $B$. melitensis $16 \mathrm{M}$ infection in the prime-boost regimen is comparable to the $B$. melitensis Rev.1 commercial vaccine in mouse and guinea pig models. This study is a substantial step for the development of a safe and protective human brucellosis vaccine.

\section{Data Availability}

All datasets generated for this study are included in the article/supplementary material. 


\section{Conflicts of Interest}

No potential conflict of interest was reported by the authors.

\section{Authors' Contributions}

KT and ShR planned and designed the present work. NZ, ShR, DB, NA (in guinea pig model), and MS were responsible for realizing the work. DB and KT were responsible for the data acquision and analysis. DB drafted and revised the manuscript. KT and ZhK approved the final version of the manuscript. All authors read and approved the final manuscript. ZhK and KT contributed equally to this work and are corresponding authors.

\section{Acknowledgments}

The authors express their gratitude to B. Yespembetov, Y. Kozhamkulov, and D. Inkarbekov, employees of the Research Institute for Biological Safety Problems, for their assistance in the present study. This work was supported by the Science Committee of the Ministry of Education and Science of the Republic of Kazakhstan (Grant No. AP05131463).

\section{Supplementary Materials}

Figure S1: insertion of Brucella proteins in the NS1 gene as determined by RT-PCR upon accumulation in CE. (Supplementary Materials)

\section{References}

[1] E. M. Galińska and J. Zagórski, "Brucellosis in humans-etiology, diagnostics, clinical forms," Annals of Agricultural and Environmental Medicine, vol. 20, no. 2, pp. 233-238, 2013.

[2] G. Pappas, N. Akritidis, M. Bosilkovski, and E. Tsianos, "Brucellosis," The New England Journal of Medicine, vol. 352, no. 22, pp. 2325-2336, 2005.

[3] M. Tekle, M. Legesse, B. M. Edao, G. Ameni, and G. Mamo, "Isolation and identification of Brucella melitensis using bacteriological and molecular tools from aborted goats in the Afar region of North-Eastern Ethiopia," BMC Microbiology, vol. 19, no. 1, p. 108, 2019.

[4] W. Ahmed, K. Zheng, and Z. F. Liu, "Establishment of chronic infection: Brucella's stealth strategy," Frontiers in Cellular and Infection Microbiology, vol. 6, no. 30, 2016.

[5] J. Lalsiamthara and J. H. Lee, "Development and trial of vaccines against Brucella," Journal of Veterinary Science, vol. 18, no. S1, pp. 281-290, 2017.

[6] X. Yang, J. A. Skyberg, L. Cao, B. Clapp, T. Thornburg, and D. W. Pascual, "Progress in Brucella vaccine development," Frontiers in Biology, vol. 8, no. 1, pp. 60-77, 2013.

[7] S. D. Perkins, S. J. Smither, and H. S. Atkins, "Towards a Brucella vaccine for humans," FEMS Microbiology Reviews, vol. 34, no. 3, pp. 379-394, 2010.

[8] A. Al-Mariri, A. Tibor, P. Mertens et al., "Protection of BALB/c mice against Brucella abortus 544 challenge by vaccination with bacterioferritin or P39 recombinant proteins with CpG oligodeoxynucleotides as adjuvant," Infection and Immunity, vol. 69 , no. 8, pp. 4816-4822, 2001.
[9] M. M. Kahl-McDonagh, P. H. Elzer, S. D. Hagius et al., "Evaluation of novel Brucella melitensis unmarked deletion mutants for safety and efficacy in the goat model of brucellosis," Vaccine, vol. 24, no. 24, pp. 5169-5177, 2006.

[10] A. Ivanov, K. Salmakov, S. Olsen, and G. Plumb, "A live vaccine from Brucella abortus strain 82 for control of cattle brucellosis in the Russian Federation," Animal Health Research Reviews, vol. 12, no. 1, pp. 113-121, 2011.

[11] A. Lopez-Merino, J. Asselineau, A. Serre, J. Roux, S. Bascoul, and C. Lacave, "Immunization by an insoluble fraction extracted from Brucella melitensis: immunological and chemical characterization of the active substances," Infection and Immunity, vol. 13, no. 2, pp. 311-321, 1976.

[12] K. A. Pasquevich, A. E. Ibañez, L. M. Coria et al., "An oral vaccine based on $\mathrm{U}-\mathrm{Omp} 19$ induces protection against $\mathrm{B}$. abortus mucosal challenge by inducing an adaptive IL-17 immune response in mice," PLoS One, vol. 6, no. 1, article e16203, 2011.

[13] M. V. Delpino, S. M. Estein, C. A. Fossati, P. C. Baldi, and J. Cassataro, "Vaccination with Brucella recombinant DnaK and SurA proteins induces protection against Brucella abortus infection in BALB/c mice," Vaccine, vol. 25, no. 37-38, pp. 6721-6729, 2007.

[14] G. Gupta, G. Radhakrishnan, J. Harms, and G. Splitter, "Invasive Escherichia coli vaccines expressing Brucella melitensis outer membrane proteins 31 or 16 or periplasmic protein BP26 confer protection in mice challenged with B. melitensis," Vaccine, vol. 30, no. 27, Article ID PMC3361596, pp. 40174022, 2012.

[15] Y. He, R. Vemulapalli, and G. G. Schurig, "Recombinant Ochrobactrum anthropi expressing Brucella abortus $\mathrm{Cu}, \mathrm{Zn}$ superoxide dismutase protects mice against B. abortus infection only after switching of immune responses to Th1 type," Infection and Immunity, vol. 70, no. 5, pp. 2535-2543, 2002.

[16] D. Sáez, I. Guzmán, E. Andrews, A. Cabrera, and A. Ońate, "Evaluation of Brucella abortus DNA and RNA vaccines expressing $\mathrm{Cu}-\mathrm{Zn}$ superoxide dismutase (SOD) gene in cattle," Veterinary Microbiology, vol. 129, no. 3-4, pp. 396-403, 2008.

[17] E. Andrews, P. Salgado, H. Folch, and A. Oñate, "Vaccination with live Escherichia coli expressing Brucella abortus $\mathrm{Cu} / \mathrm{Zn}$ superoxide-dismutase: II. Induction of specific CD8+ cytotoxic lymphocytes and sensitized CD4+ IFN-gammaproducing cell," Microbiology and Immunology, vol. 50, no. 5, pp. 389-393, 2006.

[18] J. S Harms, M. A. Durward, D. M. Magnani, and G. A. Splitter, "Evaluation of recombinant invasive, non-pathogenic Eschericia coli as a vaccine vector against the intracellular pathogen, Brucella," Journal of Immune Based Therapies and Vaccines, vol. 7, no. 1, Article ID PMC2633335, p. 1, 2009.

[19] Z. Zhao, M. Li, D. Luo et al., "Protection of mice from Brucella infection by immunization with attenuated Salmonella enterica serovar typhimurium expressing A L7/L12 and BLS fusion antigen of Brucella," Vaccine, vol. 27, no. 38, pp. 5214-5219, 2009.

[20] D. Sáez, P. Fernández, A. Rivera, E. Andrews, and A. Oñate, "Oral immunization of mice with recombinant Lactococcus lactis expressing $\mathrm{Cu}, \mathrm{Zn}$ superoxide dismutase of Brucella abortus triggers protective immunity," Vaccine, vol. 30, no. 7, pp. 1283-1290, 2012.

[21] A. A. Oñate, R. Vemulapalli, E. Andrews, G. G. Schurig, S. Boyle, and H. Folch, "Vaccination with live Escherichia coli expressing Brucella abortus $\mathrm{Cu} / \mathrm{Zn}$ superoxide dismutase protects mice against virulent B. abortus," Infection and Immunity, vol. 67, no. 2, pp. 986-988, 1999. 
[22] K. A. Pasquevich, S. M. Estein, C. G. Samartino et al., "Immunization with recombinant Brucella species outer membrane protein Omp16 or Omp19 in adjuvant induces specific CD4 + and CD8+ T cells as well as systemic and oral protection against Brucella abortus infection," Infection and Immunity, vol. 77, no. 1, pp. 436-445, 2009.

[23] P. A. Rice and T. A. Steitz, "Ribosomal protein L7/L12 has a helix-turn-helix motif similar to that found in DNA-binding regulatory proteins," Nucleic Acids Research, vol. 17, no. 10, pp. 3757-3762, 1989.

[24] C. Muñoz-Montesino, E. Andrews, R. Rivers et al., "Intraspleen delivery of a DNA vaccine coding for superoxide dismutase (SOD) of Brucella abortus induces SOD-specific CD4+ and CD8+ T cells," Infection and Immunity, vol. 72, no. 4, pp. 2081-2087, 2004.

[25] K. Tabynov, "Influenza viral vector based Brucella abortus vaccine: a novel vaccine candidate for veterinary practice," Expert Review of Vaccines, vol. 15, no. 10, pp. 1237-1239, 2016.

[26] C. C. Sreenivasan, M. Thomas, R. S. Kaushik, D. Wang, and F. Li, "Influenza A in bovine species: a narrative literature review," Viruses, vol. 11, no. 6, p. 561, 2019.

[27] T. T. Hien, M. de Jong, and J. Farrar, "Avian influenza-a challenge to global health care structures," The New England Journal of Medicine, vol. 351, no. 23, pp. 2363-2365, 2004.

[28] E. Hoffmann, G. Neumann, Y. Kawaoka, G. Hobom, and R. G. Webster, "A DNA transfection system for generation of influenza A virus from eight plasmids," Proceedings of the National Academy of Sciences of the United States of America, vol. 97, no. 11, pp. 6108-6113, 2000.

[29] K. Tabynov, A. Sansyzbay, Z. Kydyrbayev et al., "Influenza viral vectors expressing the Brucella OMP16 or L7/L12 proteins as vaccines against B. abortus infection," Virology Journal, vol. 11, no. 1, p. 69, 2014.

[30] G. G. Schurig, N. Sriranganathan, and M. J. V, "Brucellosis vaccines: past, present and future," Veterinary Microbiology, vol. 90, no. 1-4, pp. 479-496, 2002.

[31] F. Roth, J. Zinsstag, D. Orkhon et al., "Human health benefits from livestock vaccination for brucellosis: case study," Bulletin of the World Health Organization, vol. 81, no. 12, pp. 867-876, 2003.

[32] S. Baloglu, T. Toth, G. Schurig, N. Sriranganathan, and S. Boyle, "Humoral immune response of $\mathrm{BALB} / \mathrm{c}$ mice to a vaccinia virus recombinant expressing Brucella abortus GroEL does not correlate with protection against a B. abortus challenge," Veterinary Microbiology, vol. 76, no. 2, pp. 193-199, 2000.

[33] S. Baloglu, S. M. Boyle, R. Vemulapalli, N. Sriranganathan, G. G. Schurig, and T. E. Toth, "Immune responses of mice to vaccinia virus recombinants expressing either Listeria monocytogenes partial listeriolysin or Brucella abortus ribosomal L7/L12 protein," Veterinary Microbiology, vol. 109, no. 1-2, pp. 11-17, 2005.

[34] R. Vemulapalli, S. Cravero, C. L. Calvert et al., "Characterization of specific immune responses of mice inoculated with recombinant vaccinia virus expressing an 18-kilodalton outer membrane protein of Brucella abortus," Clinical and Diagnostic Laboratory Immunology, vol. 7, no. 1, pp. 114-118, 2000.

[35] G. Z. Lin, J. T. Yang, S. C. Wei, S. E. Chen, S. D. Huo, and Z. R. $\mathrm{Ma}$, "Immunogenicity of adenovirus and DNA vaccines coexpressing P39 and lumazine synthase proteins of Brucella abortus in BALB/c mice," Tropical Animal Health and Production, vol. 50, no. 5, pp. 957-963, 2018.
[36] A. Cabrera, D. Sáez, S. Céspedes, E. Andrews, and A. Oñate, "Vaccination with recombinant Semliki Forest virus particles expressing translation initiation factor 3 of Brucella abortus induces protective immunity in BALB/c mice," Immunobiology, vol. 214, no. 6, pp. 467-474, 2009.

[37] A. A. Onate, G. Donoso, G. Moraga-Cid, H. Folch, S. Céspedes, and E. Andrews, "An RNA vaccine based on recombinant Semliki Forest virus particles expressing the $\mathrm{Cu}$, $\mathrm{Zn}$ superoxide dismutase protein of Brucella abortus induces protective immunity in BALB/c mice," Infection and Immunity, vol. 73, no. 6, pp. 3294-3300, 2005.

[38] K. Tabynov, Z. Kydyrbayev, S. Ryskeldinova et al., "Novel influenza virus vectors expressing Brucella L7/L12 or Omp16 proteins in cattle induced a strong T-cell immune response, as well as high protectiveness against B. abortus infection," Vaccine, vol. 32, no. 18, pp. 2034-2041, 2014.

[39] K. Tabynov, B. Yespembetov, S. Ryskeldinova et al., "Primebooster vaccination of cattle with an influenza viral vector Brucella abortus vaccine induces a long-term protective immune response against Brucella abortus infection," Vaccine, vol. 34, no. 4, pp. 438-444, 2016.

[40] R. P. Kamal, J. M. Katz, and I. A. York, "Molecular determinants of influenza virus pathogenesis in mice," Current Topics in Microbiology and Immunology, vol. 385, pp. 243-274, 2014.

[41] M. D. Tate, D. L. Pickett, N. van Rooijen, A. G. Brooks, and P. C. Reading, "Critical role of airway macrophages in modulating disease severity during influenza virus infection of mice," Journal of Virology, vol. 84, no. 15, pp. 7569-7580, 2010.

[42] H. Ueki, I.-H. Wang, S. Fukuyama et al., "In vivo imaging of the pathophysiological changes and neutrophil dynamics in influenza virus-infected mouse lungs," Proceedings of the National Academy of Sciences of the United States of America, vol. 115, no. 28, pp. E6622-E6629, 2018.

[43] W. Pan, Z. Dong, F. Li et al., "Visualizing influenza virus infection in living mice," Nature Communications, vol. 4, no. 1, 2013.

[44] I. M. Belyakov and J. D. Ahlers, "What role does the route of immunization play in the generation of protective immunity against mucosal pathogens?," Journal of Immunology, vol. 183, no. 11, pp. 6883-6892, 2009.

[45] M. J. Grilló, J. M. Blasco, J. P. Gorvel, I. Moriyón, and E. Moreno, "What have we learned from brucellosis in the mouse model?," Veterinary Research, vol. 43, no. 1, p. 29, 2012.

[46] J. A. Richt and A. García-Sastre, "Attenuated influenza virus vaccines with modified NS1 proteins," Current Topics in Microbiology and Immunology, vol. 333, pp. 177-195, 2009.

[47] E. D. Kilbourne, "Influenza pandemics of the 20th century," Emerging Infectious Diseases, vol. 12, no. 1, pp. 9-14, 2006.

[48] T. Watanabe, T. A. Bartrand, T. Omura, and C. N. Haas, "Dose-response assessment for influenza A virus based on data sets of infection with its live attenuated reassortants," Risk Analysis, vol. 32, no. 3, pp. 555-565, 2012. 